\title{
El diseño y uso \\ de indicadores de comunicación en las organizaciones antioqueñas
}

José Luis Vahos Montoya*

Recibido: 3 de abril de 2009

Aprobado: 27 de abril de 2009

\begin{abstract}
RESUMEN
Este artículo presenta resultados de la investigación Diseño de sistema de indicadores de gestión de comunicación organizacional, realizada por el Grupo de Investigación en Gestión de la Comunicación de la Facultad de Comunicación Social de la UPB. Los indicadores de gestión se han comenzado a mirar desde la comunicación organizacional para argumentar el peso estratégico que tiene ésta al interior de las organizaciones. En este artículo se hace una revisión del estado del arte sobre los indicadores de comunicación en el entorno académico antioqueño. Para tal fin se contextualiza sobre el rol de la comunicación organizacional, posteriormente se hace un acercamiento a lo que implica medir la comunicación y el artículo termina con una consulta realizada a estudiosos y consultores que vienen trabajando sobre esta temática. Ellos dan cuenta, desde su conocimiento y experiencia, de lo que se mide, la manera como se está haciendo y el grado de incursión en el desarrollo de sistemas de medición de los procesos comunicativos en las organizaciones de esta región colombiana.
\end{abstract}

Palabras clave: Medición, comunicación organizacional, indicadores de gestión, organizaciones antioqueñas.

Comunicador Social - Periodista. Grupo de Investigación en Gestión de la Comunicación. Universidad Pontificia Bolivariana. Correo electrónico: vahos911@gmail.com 


\title{
Design and use of indicators of communication in organizations of Antioquia
}

\begin{abstract}
Management indicators have begun to look from the organizational communication in order to argue the strategic weight they have inside the companies. This article is a review of the state of the art of the indicators of communication in the Antioquia academic surroundings. For this purpose it contextualizes the role of communication in organizations, then it makes an approach which involves measuring communication and the article concludes with a survey to the experts in this field. They realize from their knowledge and experience, which is measured the way how it's been doing and the level of incursion in the development of measurement systems for organizational communication into organizations of Antioquia.
\end{abstract}

Keywords: Measurement, organizational communication, management indicators, organizations of Antioquia. 


\section{INTRODUCCIÓN}

Cada vez es más frecuente escuchar que la comunicación juega un papel estratégico en las organizaciones. Hoy en día parece ser una premisa avalada por la academia y por los propios comunicadores que egresan de las facultades de comunicación colombianas. Éste es, sin duda, un logro del posicionamiento de la comunicación organizacional en el medio. Sin embargo, llevar esta tarea a la práctica no ha sido fácil, en parte debido a que aún hoy, como afirma Huerta (1997):

Existen prejuicios respecto de las potencialidades y beneficios que ofrece la comunicación (...) Las razones de este 'escepticismo' son diversas, entre ellas (...) la que se refiere a la demora en obtener resultados tangibles expresados en términos de rentabilidad económica, y la otra se funda en el excesivo esfuerzo que se requiere en tiempo y personal para impulsar (...) actividades de comunicación empresarial (Huerta, 1997, p. 51).

Resulta paradójico, entonces, que la comunicación en las organizaciones y universidades se esté analizando en términos de roles estratégicos pero su praxis no supere, en el mejor de los casos, el estadio de lo táctico y, en muchas ocasiones, se reduzca a lo meramente operativo. En este sentido, es importante aclarar que la comunicación en las empresas debe incluir en su quehacer acciones estratégicas, tácticas y operativas. El peligro radica en encasillarse en una de ellas, principalmente en la de menor rango, si bien ello no significa que sea la menos importante.

Es necesario, también, delimitar los alcances del concepto "comunicación organizacional" para poder asir más fácilmente su importancia en la gestión de la organización. Encontramos entonces que esta expresión puede entenderse desde dos puntos de vista. En primer lugar, la comunicación organizacional es un fenómeno que se da de forma espontánea y natural en todas las organizaciones, indiferente de su naturaleza, tamaño o estructura. Rodríguez (2002, p. 11) asegura que "no es posible imaginar una organización sin comunicación. Bajo esta perspectiva, la comunicación organizacional es el conjunto total de mensajes que se intercambian entre los integrantes de una organización y entre ésta y los diferentes públicos que tiene en su entorno".

Desde un segundo punto de vista se ubica a la comunicación organizacional como una disciplina que estudia la forma como ésta se presenta dentro y fuera de las organizaciones. Esta doble condición, de fenómeno y disciplina, enmarca el quehacer del comunicador organizacional. Queda claro entonces que la comunicación se presenta aunque no haya comunicadores (fenómeno), pero su efectiva gestión se logra cuando la organización cuenta con estos profesionales (disciplina).

En este artículo se indaga por el rol que juegan las comunicaciones en las entidades de Antioquia; también se trata de identificar qué procesos de comunicación adelantan y cuál es el método que siguen para medirlos. Las respuestas a estas preguntas dan cuenta de una realidad que habla del grado en que se miden las acciones comunicativas en las organizaciones de la región. Para llegar a este punto se hace primero un breve seguimiento histórico a la incorporación de la comunicación organizacional como profesión y se examina el impacto que ésta ha tenido en el entorno.

\section{LA PROFESIONALIZACIÓN DEL OFICIO DE COMUNICAR}

La comunicación organizacional tuvo que esperar que coincidieran muchos elementos para que fuese visible. El impulsador del desarrollo del entorno de la comunicación pública fue el reconocimiento progresivo del derecho universal a la información (Sotelo, 2004). Desde sus primeras manifestaciones, en la sociedad norteamericana 
de finales del siglo XIX y principios del siglo XX, se puso en la palestra la importancia de la información en las empresas cuando la libertad para crear entidades informativas sirvió para denunciar abusos y arbitrariedades de los grandes emporios económicos en expansión, y para que estos se defendieran y proyectaran una imagen positiva en los medios de comunicación.

A partir de esa situación se empezaron a perfilar campos de estudio y desempeño como las relaciones públicas, las relaciones con los medios y la publicidad. Esta expansión de dimensiones comunicativas obligó a que muchas empresas incorporaran a expertos en tales oficios para que se hicieran responsables de las comunicaciones en sus negocios. Así se comenzaron a gestar las primeras direcciones de comunicación, que más adelante, gracias a la complejidad del oficio, tomarían la forma de departamentos.

A la par de estos desarrollos, en Europa Occidental ya se fraguaban las primeras fisuras ideológicas y políticas que devinieron en el desarrollo de la Segunda Guerra Mundial. En esta contienda, la comunicación jugó un papel protagónico en lo político e ideológico. Años más tarde, técnicas como las desarrolladas por los agentes de prensa y prácticas como la publicity se fueron incorporando lentamente a la vida de las organizaciones, las cuales, poco a poco, consolidaron los departamentos de comunicaciones y publicidad hasta el punto de exportar sus experiencias y modelos a otros países (Sotelo, 2001).

La profesionalización de las relaciones públicas y la comunicación corporativa fue posible gracias a las contribuciones de los teóricos y estudiosos de aquella serie de fenómenos. Autores como Ivy Lee y Eduard Bernays fueron algunos de los soportes teóricos de las primeras tres décadas del siglo XX, así como Lucien Matrat y James E. Gruning a finales de los años sesenta del mismo siglo. De forma paralela, la conformación de asociaciones profesionales de la comunicación como la PRSA (Public Relations Society of America), en 1947, la IPRA (International Public Relation Association), en 1955 y la IABC (Internacional Association of Business Communicator), en 1970 contribuyeron a que se consolidaran teorías, conceptos y perfiles profesionales, hasta pasar de un simple oficio, a una profesión con soporte académico y gremial.

Desde entonces, modelos administrativos y de comunicación han sido exportados y adaptados a las organizaciones de todo el mundo, en especial los provenientes de Estados Unidos. Sin embargo, en la década de los ochenta surgió en Francia un nuevo modelo en la gestión de la comunicación organizacional: se trataba de la teoría Dircom.

La Dircom, o Dirección de la Comunicación, defiende la idea de que exista una unidad central de comunicaciones que se encargue de las comunicaciones internas, las relaciones públicas y la publicidad en cabeza de un jefe o "Dircom" inscrito en la alta dirección, quien es el encargado de diseñar la estrategia integral de comunicación que deberá proyectar la empresa.

Esta práctica nace como respuesta a la disyuntiva generada por la desintegración de los lenguajes corporativos entre la publicidad, las comunicaciones internas y las relaciones públicas, lo que ocasionaba que la organización emitiera tres tipos de discursos, muchas veces sin tener coherencia entre sí. La Dircom pretende, entonces, coordinar estos mensajes y lenguajes para actuar en concordancia comunicativa. Una visión similar se propuso desde los estudios de Comunicación corporativa. Van Riel (1997), representante de esta corriente, explica que

Hoy, las organizaciones son muy conscientes del peligro de la comunicación fragmentada. Temen la trascendencia de estos 'incidentes', que podrían afectar su reputación, y desean prevenir que la eficacia y 
le eficiencia del esfuerzo de la comunicación integral esté en peligro (Van Riel, 1997, p. 3).

Algunos teóricos e investigadores como Garrido, Costa y el mismo Van Riel soportan este modelo con el desarrollo de investigaciones y textos académicos que lo definen y validan. En síntesis, se pueden evidenciar tres grandes características que debe tener el Dircom: "Profesional con conocimientos y experiencia en comunicación corporativa. Capaz de traducir sus acciones a un lenguaje financiero. Capaz de diseñar, implementar (o supervisar) y controlar la estrategia de comunicaciones de la compañía" (Garrido, 2001, p. 113). Del primero y el tercer ítem se encargan las facultades de comunicación social y la experiencia laboral en el ejercicio de la profesión, pero el segundo aspecto sigue siendo el talón de Aquiles del comunicador organizacional, pues es allí donde empiezan a generarse cuestionamientos; entre ellos: ¿cómo medir los impactos que generan las acciones de comunicación en las empresas? ¿Qué parte de la suma total de la productividad corresponde a las estrategias propias y de apoyo desarrolladas desde comunicaciones? ¿Es posible posicionar la comunicación organizacional sin tener claridad acerca de cuál es su participación real en el desarrollo de las entidades?

Preguntas como estas deben responderse si se pretende alcanzar mayores niveles de posicionamiento y un rol más estratégico por parte de cada organización.

\section{MEDICIONES EN COMUNICACIÓN}

En términos generales, la medición se ha asociado a la idea de ejercer control sobre algún factor específico: un proceso, un procedimiento, una actividad y, de hecho, es ésta realmente su esencia. Sin embargo, este concepto en su acepción más tradicional ha servido más para buscar culpables, que como una oportunidad para mejorar los procesos en el interior de la empresa. (Domínguez, 2001). Esta visión -hoy en día desmitificada por la gestión por procesos, en cuanto es el proceso la falla y no el individuo-, en ocasiones, se convierte en una dificultad que debe sortearse antes de implementar sistemas de medición en las empresas.

Ahora bien, para acercarse a lo que implica medir las comunicaciones y de acuerdo con el Diccionario de Relaciones Públicas, Medición e Investigación, (Don W., 2006) medir es una forma de dar a una actividad una dimensión precisa de sus características o estado, generalmente como resultado de compararla frente a un estándar; usualmente se hace de una manera cuantificable o numérica. De forma complementaria, Beltrán (2002, p. 54) considera que la medición "consiste en determinar fuentes de información, frecuencia de medición, presentación de la información, asignación de responsables de la recolección, tabulación, análisis y presentación de la información".

Este modelo administrativo, basado en el seguimiento y el control, puede adaptarse a la comunicación en las organizaciones. De hecho, así suele hacerse en la mayoría de las entidades en las que las comunicaciones buscan dar cuenta de sus acciones para permear escenarios más estratégicos y tácticos. Pese a esto, aún no es clara la forma como se debe implementar este modelo, en parte, debido a la escasa bibliografía disponible en nuestro medio sobre la generación de indicadores de gestión desde la comunicación y para las comunicaciones y, por otro lado, debido a la diversidad de organizaciones, en términos de tipo y tamaño, lo que dificulta la generación de estándares en la medición.

El levantamiento de procesos en las empresas que, de forma voluntaria, se acogen a la implementación de sistemas de gestión de la calidad, propone de antemano la estandarización de una serie de actividades, procedimientos y procesos que varían dependiendo de la naturaleza de cada 
empresa, con la finalidad de tener soportes unificados a partir de los cuales poder hacer seguimientos y comparaciones. Hacia allí debe apuntar la comunicación en las entidades, bien sea que estén o no inscritas en sistemas de gestión de calidad.

Si bien, la norma NTC ISO 9001:2008 de gestión de la calidad no describe de forma específica el papel de la comunicación, su importancia puede inferirse cuando menciona en uno de sus apartes que "la alta dirección debe asegurarse de que se establecen los procesos de comunicación apropiados dentro de la organización y que la comunicación se efectúa considerando la eficacia del sistema de gestión de la calidad" (Icontec, 2008, p. 6).

Habrá que agradecer entonces, que, dentro de la rigurosidad de la norma, las comunicaciones no estén sujetas o predispuestas a modelos establecidos, lo que deja un amplio campo de acción para el ejercicio de la comunicación organizacional, siempre y cuando aporte al cumplimiento del sistema de gestión de la calidad o a los objetivos empresariales. Además, menciona de forma textual el establecimiento de unos procesos de comunicación, pero no señala cuáles. De ahí se desprende la tarea de cada unidad, departamento o área de comunicación de establecer sus propios procesos y garantizar la forma de medirlos y optimizarlos. Se habla, entonces, del establecimiento de indicadores de gestión en comunicaciones, definidos como el "referente cualitativo o cuantitativo del éxito de una gestión comunicacional, que ayuda a determinar el grado en que se han cumplido los objetivos de comunicación, alcanzando los resultados, y gestionando los productos de manera eficaz" (Caicedo, 2008, p. 3).

Para tratar de hilvanar el contexto de los procesos en el interior de las organizaciones, se partirá a continuación de unas precisiones conceptuales. En ese orden de ideas, y de acuerdo con la guía básica para documentar y caracterizar procesos de la Universidad Nacional de Colombia
(2008, p. 3) un macro-proceso se puede definir como el "propósito, función o servicio de una entidad o dependencia, generalmente establecido por la norma de creación de la misma"; se hace referencia a su razón de ser. Cada macro-proceso está constituido por una serie de procesos que, en suma, contribuyen al cumplimiento de ese propósito fijado.

Continuando en escala descendente, se encuentran los procesos, cuyas definiciones pueden variar pero su esencia se puede resumir como esa "actividad que utiliza recursos, y que se gestiona con el fin de permitir la transformación de elementos de entrada (inputs) en resultados (outputs) (...) Frecuentemente el resultado de un procesos constituye, de forma directa, el elemento de entrada del siguiente proceso" (ICONTEC, 2000, p. 2). Es importante resaltar que lo más apropiado es determinar cuáles son esas actividades que tienen mayor impacto sobre el proceso y establecer en ellas las mediciones, pues es cierto que todo proceso puede y debe medirse, sin embargo, no es muy práctico medirlo todo (Rincón, 1998).

Finalmente, es preciso identificar los procedimientos como aquella "forma específica para llevar a cabo una actividad o un proceso" (Arango D., et al., 2004, p. 36). En esos procedimientos se imparte una serie de pasos a seguir de manera lógica y ordenada, describiendo cómo se hace el trabajo y cómo se deben desempeñar las funciones, aspectos que varían de una organización a otra.

\section{USO Y APLICACIÓN DE INDICADORES A LOS PROCESOS DE COMUNICACIÓN EN ORGANIZACIONES ANTIOQUEÑAS}

Si bien los indicadores no son un tema reciente en la administración, la implementación de éstos en la comunicación organizacional está 
todavía en pleno desarrollo y experimentación. Bien sea desde el conocimiento empírico o desde la práctica consciente de una teoría administrativa, los indicadores de gestión han permeado las comunicaciones en las empresas de la ciudad de Medellín y del departamento de Antioquia. ¿En qué punto se encuentra este desarrollo? Es la inquietud central que se intenta resolver en este apartado.

Se ha consultado a dos expertos y académicos, en el campo de la comunicación organizacional, para conocer sus percepciones sobre el estado actual de la medición de las comunicaciones en el plano local. Se acude a estas fuentes debido a que aún no se han sistematizado ni documentado estas experiencias, tarea que empieza a realizarse en nuestro medio, de manera lenta y paulatina.

De acuerdo con Solángel Ardila, comunicadora social, experta en el área de gestión y consultora empresarial, la comunicación ha trascendido a un espacio más estratégico en las empresas, y los comunicadores se han dado cuenta de la importancia de medir los procesos, eventos, medios y espacios de comunicación a los que amerite definirles unos resultados. Para ella, la comunicación organizacional es uno de los estadios profesionales que más dificultad tiene para traducir en cifras sus aportes a las organizaciones. Los medios audiovisuales cuentan con sistemas de rating al igual que la radio; los nuevos medios cuentan con contadores de visitantes y transacciones efectivas y la prensa escrita cuenta con unos sistemas más refinados aún para costear sus publicaciones. Si bien estos sistemas de métrica no incluyen mediciones de impacto, sí suponen de antemano la intención de establecer parámetros evaluativos de las acciones de comunicación. Es preciso aclarar que sólo se mencionan estos escenarios para ejemplificar, lo que no quiere decir que la praxis de la comunicación se reduzca solamente a ellos.
¿Qué dificultades existen a la hora de medir las acciones comunicativas en las organizaciones antioqueñas? David Hernández, comunicador social, PhD en Psicología de las Organizaciones y del Trabajo, y docente universitario, considera que es difícil adaptar el tema de los indicadores al proceso comunicativo debido a que el diseño y aplicación de los indicadores de gestión provienen de la administración y las finanzas. De acuerdo con él, es claro que existen deficiencias en la formación administrativa de los comunicadores organizacionales, exceptuando a aquellos profesionales que han profundizado en estos temas, bien sea porque han realizado investigaciones 0 han estudiado postgrados en la materia.

Existe, sin embargo, otro factor que ha impedido la práctica efectiva de esta herramienta de gestión. Infortunadamente, no todas las organizaciones para las que trabajan los comunicadores operan bajo el marco de la medición de sus resultados. Claro está que esto no es en sí una resistencia a medir y a buscar una gestión efectiva; más bien, de fondo sigue reflejando el poco conocimiento que tienen las organizaciones sobre la comunicación. Prueba de ello es que lo que se está midiendo en algunas entidades de Medellín y Antioquia sigue siendo muy experimental e incipiente, es decir, se ha limitado en la mayoría de los casos a hacer seguimiento numérico de actividades, asistencias, publicaciones, free press, lectura de medios, cumplimiento y ejecución de actividades (Ardila S., 2008), que si bien miden aspectos básicos, sirven de punto de referencia para generar la medición de aspectos más complejos. En otras palabras, estos indicadores, también conocidos como de cumplimiento, sirven para demostrar que se ha hecho lo que se planificó hacer, sin embargo, las mediciones en comunicación han evolucionado y ahora se busca evaluar otros aspectos. Por fortuna, el creciente interés de los comunicadores sobre cuestiones administrativas puede fortalecer esta tendencia gracias a las permanentes opciones de capacitación que ofrecen 
universidades y facultades de comunicación, así como algunas agremiaciones profesionales.

Partiendo de la descomposición de la expresión "indicador de gestión", gestionar significa conseguir ese objetivo que se tiene (HERnándEz D., 2008). Este aspecto resulta crucial para comprender el cambio que debe tomar la medición en las comunicaciones; se habla entonces, de medir el impacto de la comunicación. Así, ya no se debe cuestionar lo que se ha hecho sino sobre lo que se ha gestionado, es decir, lo conseguido en las labores que ya se han ejecutado.

En la región, esa transición de la métrica comunicacional del cumplimiento al impacto ha sido lenta, en parte, debido a que percibir un cambio generado por una acción en comunicaciones puede no ser inmediato y, por el contrario, tardarse un tiempo considerable en manifestar los efectos esperados o no. Así mismo, y pese a la escasa bibliografía existente en el medio, la academia empieza a estudiar rigurosamente este ámbito en la comunicación organizacional y espera diseñar la forma más adecuada para hacer este tránsito, teniendo en cuenta la idiosincrasia de las organizaciones de la región.

Un aspecto importante en la medición comunicacional es el levantamiento de los procesos de comunicación como una metodología práctica que ayuda a estandarizar las actividades y procedimientos para facilitar su indexación. Al respecto, tres estadios de la administración de empresas han servido de insumos para el desarrollo de esta tarea: a) la planeación estratégica, b) la implementación de sistemas de gestión de la calidad y c) el control interno. Estas tres figuras, de forma separada o conjunta, han creado la necesidad de evaluar el cumplimiento más que el alcance, pero su importancia está en facilitar la identificación de unas actividades macro que son comunes a las áreas de comunicación de casi todas las organizaciones: comunicación interna, comunicación externa, administración de eventos, publicaciones y medios de comunicación... es lo que se conoce como los procesos de comunicación. Es importante aclarar que éstos pueden adoptar un mismo nombre y parecer iguales en diversas entidades, pero bajo los lineamientos de la dirección y el modus operandi, pueden variar significativamente de una organización a otra. Por eso, la generación de un estándar absoluto no deja de ser utópica hasta el momento.

En Antioquia, y de acuerdo con la opinión de los consultores citados, los procesos de comunicaciones más comunes en las empresas son: la gestión de la imagen corporativa, la divulgación de la plataforma estratégica, la administración de medios de comunicación, la gerencia de eventos, la comunicación interna y con steakholders externos al igual que el posicionamiento de marca. Sin embargo, las áreas de comunicación constituyen un apoyo transversal a todas las divisiones del organigrama, lo cual quiere decir que su trabajo no se limita exclusivamente a responder por sus procesos sino también ayuda a gestionar los de otras áreas en las cuales tiene una incidencia indirecta. Es lo que se conoce como procesos de apoyo. Es evidente la participación de las comunicaciones en procesos de gestión humana como: inducción, reinducción, formación, capacitación, entrenamiento, clima e identidad organizacional; en mercadeo: promoción, publicidad, comunicación directa, mercadeo relacional, administración de bases de datos, publicaciones; en la Dirección: protocolo empresarial, divulgación de la plataforma y de la dirección estratégica, y en planeación, al apoyar los procesos de prospectiva organizacional. Esto, sin contar las unidades de negocio a las cuales se prestan asesorías, soportes informativos y estrategias de comunicación, es decir, lo que se conoce como el cliente interno de las comunicaciones en la organización.

Muchas veces, a la hora de sumar los esfuerzos que el área hace para el cumplimiento de los 
objetivos de la organización, estos procesos pasan inadvertidos hasta para los mismos comunicadores. En esa medida, se evidencia la necesidad de construir indicadores compartidos (Ardila, 2008) en los que se establezca de forma clara y real la participación de las comunicaciones en el cumplimiento de las metas de determinada dependencia; así, la sumatoria de esos aportes ayuda a hacer legítima la labor del área.

Una de las grandes dificultades que existe, no sólo en Antioquia sino en términos generales, a la hora de establecer procesos y diseñar una medición sobre ellos, es la definición de la variable que se debe medir para que el indicador responda de manera efectiva a lo que se requiere. Es importante, entonces, indicar que, básicamente, los indicadores se definen a partir de los objetivos de cada proceso; éstos empiezan a delimitar la variable que se requiere medir. Sin embargo, este es sólo el primer insumo necesario para determinar de manera acertada una variable. Lo segundo es que no se puede definir un indicador del cual no se tiene una fuente confiable de información y, en tercer lugar, un aspecto que es fundamental, es propiciar el establecimiento de una variable cuyo indicador sea útil para la toma de decisiones (Hernández D., 2008); de lo contrario, todo lo anterior carecerá de fundamento práctico.

Otro método para determinar la variable de comunicación que se debe medir es identificar las necesidades del perceptor del proceso, es decir, y en este caso, el usuario de la comunicación, sea interno o externo (Ardila, 2008). Estos perceptores manifiestan unas necesidades de comunicación y es ahí donde se debe definir qué es lo que ellos quieren que sea parametrizado, en otras palabras, el perceptor "dice" qué es importante o susceptible de medición. Esta lógica obedece a un esquema clásico de atención al cliente que se puede incorporar en comunicaciones a la labor de satisfacción del público interno del que se habló en otro apartado de este artículo.
Estas perspectivas de ninguna manera son excluyentes, por el contrario, se complementan y ayudan al establecimiento claro de qué aspectos se deben medir desde el levantamiento de los procesos del área de comunicaciones y desde los procesos de soporte que maneja el área frente a otras dependencias de la organización.

Otra de las dificultades que presentan las organizaciones es la medición del impacto en variables complejas como la imagen y la cultura organizacional. En este punto es conveniente hacer algunas precisiones conceptuales. Capriotti (2004, p. 62) define la imagen corporativa como "la estructura o esquema mental sobre una compañía que tienen sus públicos, integrada por el conjunto de atributos que los públicos utilizan para identificar y diferenciar a esa compañía de las demás"; en este campo, la dificultad radica en volver tangible lo que, por naturaleza, es inmaterial.

De otro lado, la cultura organizacional está constituida por "la suma de hipótesis o creencias fundamentales sobre las cuales funcionan tanto la organización cómo las personas que la integran" (Aguilera y Camacho, 2007, p. 35).

Es importante mencionar que en estas variables, el aporte de comunicaciones es fundamental y tiene una incidencia directa, sin embargo, no es el único factor que entra en juego. Áreas como Gestión Humana y la Dirección hacen parte de esta compleja estructura. Entonces, ¿cómo medir estas variables?

Estas son las llamadas variables blandas que se caracterizan por tener algún nivel de relación con la percepción, la observación o las conductas. Para establecer parámetros de medición para ellas se deben utilizar las herramientas de la psicología y la sociología (Hernández, 2008), es decir, todo lo que tiene que ver con estudios de opinión y percepción, entrevistas, encuestas y cuestionarios, sólo por citar algunas de estas herramientas. En 
ellas, la ponderación o calificación cuantitativa de factores cualitativos, ayudan a establecer indicadores en estas variables complejas.

En esa misma línea y retomando el esquema de atención al cliente o usuario, un prerrequisito para abordar este tipo de variables es la parametrización, (Ardila, 2008) efecto que se logra "pesándolas," dependiendo de lo que los usuarios manifiesten que es importante medir. En otras palabras, se trata de ponderar, asignar un valor numérico, de acuerdo con la importancia o relevancia que le asigne la persona consultada. Así pues, la formulación del indicador saldrá del resultado de las respuestas de los clientes o usuarios en los diagnósticos de comunicaciones y en los inventarios de necesidades (lo que el cliente interno o externo necesita o requiere del área de comunicaciones).

La serie de factores hasta aquí enunciados explica la dificultad que presentan las entidades antioqueñas para levantar y estructurar este tipo de indicadores, sin embargo, la tendencia está cambiando paulatinamente. Cada vez son más las instituciones que en el plano regional y local implementan sistemas de medición en sus comunicaciones, tal es el caso del Hospital Universitario San Vicente de Paúl, la empresa ensambladora de vehículos Sofasa, la Alcaldía de Sabaneta, el Grupo Regional ISO de la Universidad de Antioquia, Corpaúl, ISA, EPM, Noel, Olasa, la Compañía Nacional de Chocolates y el Hospital General de Medellín, entre otras.

\section{CONCLUSIÓN}

En este artículo se ha presentado, de forma sucinta, la manera en que gradualmente, la comunicación organizacional ha cobrado importancia, dada la cantidad de escenarios en los que se desenvuelve y contribuye a la organización. Como parte de ese ejercicio, se ha mencionado la necesidad de medir y justificar las acciones que se desarrollan desde el área, lo cual es posible mediante la implementación y uso de los indicadores de gestión adecuados a este tipo de procesos.

A partir de la opinión de expertos en el tema, se han podido evidenciar las dificultades y alcances de la medición de las acciones comunicativas en el entorno antioqueño. Uno de los aspectos que más llama la atención es la poca bibliografía existente desde la óptica comunicacional que, sumado a la baja formación de los comunicadores en asuntos administrativos y financieros, dificulta el uso y la aplicación de mediciones de la comunicación en muchas organizaciones.

De otro lado, se evidencia que las mediciones, en términos generales, se siguen estableciendo principalmente sobre indicadores de cumplimiento, lo cual es importante en la medida que se tenga conciencia de que no basta con medir el número de tareas ejecutadas de acuerdo con el plan, sino los efectos e impactos que se logran a partir de éstos. El tránsito es lento, pero ya se comienzan a evidenciar algunos casos que ponen de manifiesto el cambio de paradigma en la comunicación organizacional de la región.

Si bien está claro que la comunicación es un proceso transversal a toda la organización, es imperiosa la necesidad de determinar el grado en el que las acciones de comunicación aportan al desarrollo y cumplimiento de esas áreas de la organización que desde la comunicación se potencian. Esa gestión también debe medirse y de hecho se está haciendo, aunque no de manera generalizada. Asimismo, variables tan complejas como la cultura y la identidad también son susceptibles de medir desde la comunicación, pues, si bien es cierto que no es el único componente implicado es estos fenómenos organizacionales, tiene una alta incidencia en ellos y puede afectarlos favorable o desfavorablemente. Su correcta gestión y la medición de ésta resultan fundamen- 
tales a la hora de determinar el comportamiento de estas variables.

Con base en lo expuesto, se puede decir que la comunicación organizacional en las empresas de Medellín y Antioquia está comenzado a estudiar y a repensar la manera como asume su rol en el interior de las organizaciones. Parte de ese cambio lo ha promovido la generación de indicadores; si bien aún son básicos, poco a poco se comienza a investigar la manera más acertada para justificar con cifras los impactos que generan las acciones de comunicación en las distintas organizaciones.

\section{REFERENCIAS BIBLIOGRÁFICAS}

Aguilera J. y Camacho N. (2007) Gerencia integral de comunicaciones. Claves para estructurar los nuevos departamentos de comunicación. (1ª ed., p. 35) Bogotá: Ecoe ediciones.

Arango D., Arango E., Escandón H., García C., Jaramillo C., Peláez J., Pineda J., Reina R., Trejos A. y Yepes M. (2004) Seminario de Investigación Gerencia por Procesos (Innovación enfocada al cliente, un proceso para lograr la competitividad en las organizaciones) Medellín, Colombia: Universidad Pontificia Bolivariana, Escuela de Ciencias Estratégicas, Facultad de Administración.

Ardila S., entrevista personal, 23 de octubre de 2008. Medellín.

Beltrán J. (2002) Indicadores de gestión: herramientas para lograr la competitividad. (2 ed., p. 54) Bogotá: 3R.

Caicedo, G. (2008) Cómo diseñar un sistema para medir la comunicación. Manuscrito no publicado.

Capriotti, P. (2004) La imagen corporativa. Losada, J. Gestión de la Comunicación en la Organizaciones. (1ª ed., p. 62) España: Editorial Ariel S. A.

Domínguez G. (2001) Indicadores de gestión. Un enfoque sistémico. (3ª ed., p. 101) Medellín: Biblioteca Jurídica DIKE.

Don W. (2006) Dictionary of Public Relations Measurement and Research [Diccionario de Relaciones Públicas, Medición e Investigación]. Recuperado el día 18 del mes 10 del año 2008, http://www.instituteforpr.org/files/uploads/ PRMR_Dictionary.pdf

Garrido, F. (2001) La comunicación estratégica. Las claves de la comunicación empresarial del siglo XXI. (1ª Ed., p. 113) Barcelona: Gestión 2000.

Hernández, D., entrevista personal, 22 de agosto de 2008. Medellín.

Huerta, R. (1997) Comunicación y procesos de socialización empresarial. Contratexto, (11).

Icontec (2000) Norma Técnica Colombiana NTC-ISO 9004:2000. Sistemas de gestión de la calidad. Directrices para la mejora del desempeño. (2a Actualización).

Icontec (2008) Norma Técnica Colombiana NTC-ISO 9001:2008. Sistemas de gestión de la calidad. Requisitos. (3ª Actualización). 
Rincón, R. (1998) Los indicadores de gestión organizacional: una guía para su definición. Revista Universidad EAFIT (No. 111), p. 47.

Rodríguez, H. (2002) Definición y alcance de la comunicación organizacional. En: Fernández, C. La comunicación en las organizaciones ( $2^{\mathrm{a}}$ ed., p. 11-12) México: Trillas.

Sotelo, C. (2001) Introducción a la comunicación institucional. Barcelona: Ariel, S. A.

Sotelo, C. (2004) Historia de la gestión de la comunicación en las organizaciones. En: Losada, J. Gestión de la Comunicación en la Organizaciones. (1 $1^{\mathrm{a}}$ ed., p. 36) Barcelona: Ariel S.A.

Universidad Nacional de Colombia. (2008) Administración y mejoramiento del sistema de gestión. Guía básica para documentar. Caracterización de procesos. Recuperado el día 15 del mes 10 del año 2008, del sitio web de la Universidad Nacional de Colombia: http://www.simege.unal.edu.co/simege/ inal2008/GUIA_BASICA_PARA_ DOCUMENTAR

Van Riel, C. (1997) Comunicación Corporativa, (p.3) Madrid: Prentice Hall. 